\title{
Logging evaluation on mechanical-damage characteristics of the vicinity of the wellbore in tight reservoirs
}

\author{
Tianshou $\mathrm{Ma}^{1}$ D $\cdot$ Junchuan $\mathrm{Gui}^{2} \cdot$ Ping Chen ${ }^{1}$
}

Received: 10 November 2020 / Accepted: 26 May 2021 / Published online: 4 June 2021

(c) The Author(s) 2021

\begin{abstract}
The rock mechanical behavior and damage characteristic is of great importance for in situ stress evaluation, wellbore stability analysis and hydraulic fracturing design. The velocities of elastic waves are usually reduced in the presence of rock damage, it may be used for determining the progressive damage of the rock. Therefore, this paper aims to investigate the damage characteristics of transversely isotropic tight sand formation, the rock mechanical and damage parameters in the vicinity of the wellbore were calculated using acoustic logging data. The results indicated that the Poisson's ratio and damage parameters decrease with increasing in radial distance, while the elastic modulus and Thomsen's coefficients increase. At the same radial position, the vertical elastic modulus is smaller than that of the horizontal, the degree of anisotropy for P-wave is greater than that of S-wave, and the horizontal damage parameter is greater than that of the vertical, which indicated that the micro-cracks near the wellbore mainly occur in the horizontal direction. The changes in mechanical parameters, Thomsen's coefficients and damage parameters rapidly changed in the range of 1.0-1.8 times of borehole radius. The variations of Thomsen's coefficients and damage parameters in mudstone are obviously greater than that of sandstone, which may be due to the induced damage between rocks and drilling fluid of mudstone is much higher than sandstone.
\end{abstract}

Keywords Tight sandstone $\cdot$ Acoustic logging $\cdot$ Rock mechanical parameters $\cdot$ Transverse isotropy $\cdot$ Anisotropy coefficients $\cdot$ Damage parameters $\cdot$ Thomsen's coefficients

\section{Introduction}

Tight sand is a kind of very important unconventional oil and gas reservoir, it is also the highest class of unconventional oil and gas resources that developed all of the world (Zou et al. 2018). Tight sand gas is playing an increasingly important role for oil and gas supplies. Tight sand oil and gas can help us to optimize the energy consumption structure, reduce environmental pollution, and reduce the external dependence of oil and gas (Dai et al. 2012; Boosari et al. 2016). Consequently, the Chinese government is vigorously promoting exploration and development of tight sand oil and gas (Wang et al. 2012; Ming et al. 2015; Chai et al.

Tianshou Ma

matianshou@126.com; matianshou@swpu.edu.cn

1 State Key Laboratory of Oil and Gas Reservoir Geology and Exploitation, Southwest Petroleum University, Chengdu 610500, Sichuan, China

2 Research Institute of Shale Gas, PetroChina Southwest Oil and Gasfield Company, Chengdu 610051, Sichuan, China
2016). However, China's tight oil and gas is characterized by great depth, well-developed natural fractures, low porosity, low permeability, and low pore pressure, and its successful development depends on horizontal well and stimulation technology (Liu et al. 2015; Ming et al. 2015; Chai et al. 2016). In the process of drilling, wellbore breakout, wellbore caving, wellbore enlargement, borehole collapse, induced pipe sticking and other wellbore instability problems are often encountered (Luo et al. 2016; Yang et al. 2020; Małkowski et al. 2020). In the process of hydraulic fracturing, natural fractures have a significant impact on hydraulic fracture propagation, which makes hydraulic fractures passing through or tracing the natural fractures, consequently, the effects of hydraulic fracturing is affected (Guo and Gou 2015; Luo et al. 2018; Wang et al. 2020). In fact, all of these problems are related to in situ rock properties and stresses. However, due to the impact of drilling and hydraulic fracturing operations, the micro-fractures that developed in tight sand formation gradually expand, merge and penetrate to form macro-fractures, which further develop and finally break down. The changes of micro- and macro-structure of

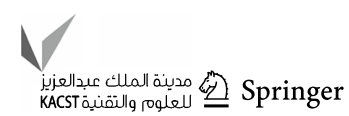


tight sand will make its rock properties, damage characteristics, in situ stresses, wellbore stability, and hydraulic fracturing change correspondingly. Acoustic logging is useful way to measure the in situ response of the formation rock in the vicinity of the wellbore (Gui et al. 2018, 2020), especially for evaluation of mechanical properties, anisotropy, damage characteristics, and their distribution characteristics (Xie et al. 2012; Xiong et al. 2021).

In fact, the damage characteristic is a most important aspect to describe the changes of rock properties (Gui et al. 2018), it can also be used for evaluation of mechanical properties, anisotropy, in situ stresses, wellbore stability, and hydraulic fracturing. In order to evaluate rock damage characteristics, the most useful way is to direct measure rock mechanical behaviors (Sayers and Kachanov 1995; Eberhardt et al. 1999; Sayers 1999; Chang and Lee 2004; Chaki et al. 2008; Ma and Chen 2014; Xue et al. 2014; Zhang et al. 2015; Ma et al. 2016; Yang et al. 2018; Zhou et al. 2019; Cheng 2019; Chu et al. 2020; Zhu et al. 2019; Liu et al. 2020; Song et al. 2020), the uniaxial compression tests, triaxial compression tests, ultrasonic wave tests, acoustic emission tests, CT scans, and NMR tests were utilized to characterize rock damage. Most of these studies indicated that rock damage is related to micro-cracking, the higher the degree of rock damage, the much more micro-cracking occurred in rock specimen. However, the damage characteristics of anisotropic rocks are seldom investigated.

Regarding the evaluation of damage characteristics of anisotropic rocks, Sayers and Kachanov (1995) investigated the changes of elastic wave and anisotropy of rock induced by micro-cracks. Mavko et al. (1995) and Sayers (1999) also investigated the changes of elastic wave and anisotropy of rock induced by micro-cracks and its stress-dependence, and they pointed out that both stress and micro-crack can induce the anisotropy of wave velocity in rocks. In fact, Schoenberg (1980) firstly proposed a simple theoretical model for predicting wave velocity for an imperfect contacted formation with two kinds of elastic mediums. On this basis, Sayers and cooperator indicated that the failure of brittle rocks during compression is preceded by the formation, growth, and coalescence of micro-cracks, these micro-cracks are not randomly oriented, which makes the rock displays an elastic anisotropy, and the elastic anisotropy due to cracks can be expressed in terms of a second-rank and fourth-rank crack density tensor (Sayers and Kachanov 1995; Sayers 1999). Sarout et al. (2007) conducted a series of triaxial compression and acoustic emission tests for shale rock, the ultrasonic wave velocity was also tested for different confining pressure, and the rock damage was finally evaluated for different confining pressure. Tang and $\mathrm{Wu}(2015)$ investigated the stress-dependent anisotropy of mudstone and shale with low porosity, and the evolution characteristics of micro-cracks and damage were also investigated. In addition, Sayers and cooperator also indicated that the elastic wave velocities are reduced in the presence of open micro-cracks and fractures (Sayers and Kachanov 1995; Sayers 1999), therefore, it may be used for monitoring the progressive damage of the rock.

Currently, the evaluation of damage characteristics of anisotropic rocks mainly focuses on the indoor experiments, the anisotropy induced by micro-cracks and stresses were also investigated by using both experimental and theoretical methods. However, the current researches are fully different to the real down-hole situation, because the rock in the vicinity of the wellbore is subjected to in situ geo-fluids, in situ stress, wellbore pressure, and operation conditions. The indoor experiment cannot reveal the distribution and evolution of rock mechanical properties, anisotropy and damage characteristics, and it also has some disadvantages, such as difficulty in coring, expensive testing and limited testing data (Ma et al. 2018). As we know, acoustic logging contains abundant formation information and in situ response during operation, and it has the advantages of relatively low cost and continuous sampling. However, the study of evaluating formation rock damage in the vicinity of the wellbore by acoustic logging data is still seldom investigated. Therefore, the present paper aims to evaluate the anisotropic damage of the formation rock in the vicinity of the wellbore by using acoustic logging data, and the paper is organized as follows: In Sect. 2, the logging calculation method of rock stiffness matrix was introduced. In Sect. 3, the logging evaluation for rock mechanics and damage parameters were presented, especially for the calculation method of radial distribution of the parameters. In Sect. 4, the logging interpretation results of DL 1 well was introduced, and the distribution of rock mechanical and damage parameters were analyzed for both sandstone and mudstone rocks, and the damage characteristics of both sandstone and mudstone rocks was compared. The results can help us understand the distribution and evolution of rock mechanical properties, anisotropy and damage characteristics in the vicinity of the wellbore, and it also can provide some theoretical basis for in situ stress evaluation, borehole stability analysis and hydraulic fracturing design.

\section{Logging calculation of the stiffness matrix}

\section{Characterization of elastic anisotropy}

For any kind of elastic medium, the elastic waves will occur when the dynamic stress does not exceed the elastic limit of the medium. The propagation characteristic of the elastic wave is related to rock dynamics and can be expressed by Hook's law (Higgins et al. 2008):

$\sigma_{i j}=C_{i j k l} \varepsilon_{k l}-\alpha P_{p}$ 
where $\sigma_{i j}$ is the stress tensor; $C_{i j k l}$ is the rock stiffness matrix; $\varepsilon_{k l}$ is the strain tensor; $\alpha$ is the Biot's coefficient; $P_{p}$ is the pore pressure; subscript $i, j, k, l=1,2,3,4,5,6$.

Due to the symmetry of stiffness matrix $\left(C_{i j k l}\right)$, the unknown elements in the stiffness matrix $\left(C_{i j k l}\right)$ can be reduced from 81 to 21, and Eq. (1) can be expressed as:

$\sigma_{i}=C_{i j} \varepsilon_{j}-\alpha P_{p}$

where

$C_{i j}=\left[\begin{array}{cccccc}C_{11} & C_{12} & C_{13} & C_{14} & C_{15} & C_{16} \\ & C_{22} & C_{23} & C_{24} & C_{25} & C_{26} \\ & C_{33} & C_{34} & C_{35} & C_{36} \\ & & C_{44} & C_{45} & C_{46} \\ & & & C_{55} & C_{56} \\ & & & & C_{66}\end{array}\right]$

where $C_{11}, C_{12}, C_{13}, C_{14}, C_{15}, C_{16}, C_{21}, C_{22}, C_{23}, C_{24}, C_{25}$, $C_{26}, C_{31}, C_{32}, C_{33}, C_{34}, C_{35}, C_{36}, C_{44}, C_{45}, C_{46}, C_{55}, C_{56}$ and $C_{66}$ are the element of the stiffness matrix.

In general, there are three kinds of situations for describing rock elastic properties, i.e., the orthotropic, transverse isotropic, and isotropic (Wang et al. 2012). For tight sand formations, the formation rock usually shows transversely isotropic characteristics, due to the horizontal sedimentary environment, and it can also be called as vertically transverse isotropic (VTI) medium. Due to the symmetry of stiffness matrix $\left(C_{i j}\right)$ in vertical direction, the element of stiffness matrix: $C_{11}=C_{22}, C_{44}=C_{55}, C_{12}=C_{21}, C_{13}=C_{31}=C_{23}=C_{32}$. Thus, the stiffness matrix $\left(C_{i j}\right)$ can be simplified as:

$C_{i j}=\left[\begin{array}{cccccc}C_{11} & C_{12} & C_{13} & 0 & 0 & 0 \\ & C_{11} & C_{13} & 0 & 0 & 0 \\ & & C_{33} & 0 & 0 & 0 \\ & & & C_{44} & 0 & 0 \\ & & & & C_{44} & 0 \\ & & & & & C_{66}\end{array}\right]$

In Eq. (4), there are some independent parameters, and these independent parameters can be determined using indoor ultrasonic wave tests (Gui et al. 2018; Huang et al. 2020):

$$
\left\{\begin{array}{l}
C_{11}=\rho V_{\mathrm{P} 11}^{2} \\
C_{33}=\rho V_{\mathrm{P} 33}^{2} \\
C_{44}=\rho V_{\mathrm{S} 33}^{2} \\
C_{66}=\rho V_{\mathrm{S} 11 b}^{2} \\
C_{13}=-C_{44}+\left[4 \rho^{2} V_{\mathrm{P} 45}^{2}-2 \rho V_{\mathrm{P} 45}^{2}\left(C_{11}+C_{33}+2 C_{44}\right)+\left(C_{11}+C_{44}\right)\left(C_{33}+C_{44}\right)\right]^{0.5}
\end{array}\right.
$$

where $\rho$ is the rock density; $V_{\mathrm{P} 11}, V_{\mathrm{P} 45}$ and $V_{\mathrm{P} 33}$ is the $\mathrm{P}$-wave velocities in the $0^{\circ}, 45^{\circ}$, and $90^{\circ}$ directions with respect to the plane of symmetry, respectively; $V_{\mathrm{S} 11 \mathrm{~b}}$ and $V_{\mathrm{S} 33}$ is the $\mathrm{S}$-wave velocities in the $0^{\circ}$, and $90^{\circ}$ directions with respect to the plane of symmetry, respectively.

\section{Logging calculation of stiffness coefficients}

In order to calculate these independent elements of stiffness matrix using acoustic logging data, we need to determine the relationship between wave velocities with acoustic logging data. In general, only three parameters of them can be obtained directly by using full wave logging data, i.e., $C_{33}, C_{44}$, and $C_{66}$ (Tang and Zheng 2004; Wang et al. 2007). Thereinto, $C_{33}$ can be determined by the P-wave logging data, $C_{44}$ can be determined by the S-wave logging data, while $C_{66}$ can be determined by Stoneley wave logging data. But the other two independent parameters can be determined using the following empirical equations (Schoenberg et al. 1996; Schoenberg and Douma 1998):

$\left\{\begin{array}{l}C_{11}=C_{33}+2\left(C_{66}-C_{44}\right) \\ C_{12}=C_{11}-2 C_{66}\end{array}\right.$

Regarding the independent parameter $C_{13}$, it is dependent on the formation type, Schoenberg and his cooperator conducted theoretical and experimental investigation (Schoenberg et al. 1996; Schoenberg and Douma 1998), and proposed a more accurate anisotropic model for calculating parameter $C_{13}$. On this basis, Sayers (2008) proposed a constraint condition for determination of parameter $C_{13}$ :

$$
\left(C_{13}+C_{33}\right)\left(C_{13}+2 C_{66}\right)=C_{33}\left(C_{13}+C_{11}\right)
$$

By solving Eq. (7), the parameter $C_{13}$ can be expressed as:

$$
C_{13}=-C_{66}+\left[C_{66}^{2}+\left(C_{11}-2 C_{66}\right) C_{33}\right]^{0.5}
$$




\section{Logging evaluation for rock mechanics and damage parameters}

\section{Anisotropic mechanical parameters and Thomsen's coefficients}

Rock mechanical parameters, such as elastic modulus and Poisson's ratio, are very important parameters for in situ stress determination, wellbore stability analysis and hydraulic fracturing design. For tight sand formations, the mechanical parameters consist of two elastic modulus $\left(E_{v}\right.$ and $\left.E_{h}\right)$ and two Poisson's ratio $\left(\mu_{v}\right.$ and $\left.\mu_{h}\right)$, and these parameters can be expressed by using rock stiffness coefficients (Walsh et al. 2006; Denney 2012):

$\left\{\begin{array}{l}\left\{\begin{array}{l}E_{v}=C_{33}-2 C_{13}^{2} /\left(C_{11}+C_{12}\right) \\ E_{h}=\left(C_{11}-C_{12}\right)\left(C_{11} C_{33}-2 C_{13}^{2}+C_{12} C_{33}\right) / C_{11} C_{33}-C_{13}^{2}\end{array}\right. \\ \left\{\begin{array}{l}\mu_{v}=C_{13} /\left(C_{11}+C_{12}\right) \\ \mu_{h}=\left(C_{12} C_{33}-C_{13}^{2}\right) /\left(C_{11} C_{33}+C_{13}^{2}\right)\end{array}\right.\end{array}\right.$

where $E_{v}$ and $E_{h}$ are elastic modulus perpendicular and parallel to the bedding plane, respectively; $\mu_{\nu}$ and $\mu_{h}$ are Poisson's ratio perpendicular and parallel to the bedding plane, respectively.

In order to characterize the degree of rock anisotropy, the Thomsen's coefficients $(\varepsilon, \gamma, \delta)$ were introduced (Thomsen 1986):

$$
\left\{\begin{array}{l}
\varepsilon=0.5\left(C_{11} / C_{33}-1\right) \\
\gamma=0.5\left(C_{66} / C_{44}-1\right) \\
\delta=0.5\left[\left(C_{13}+C_{44}\right)^{2}-\left(C_{33}-C_{44}\right)^{2}\right] / C_{33}\left(C_{33}-C_{44}\right)
\end{array}\right.
$$

\section{Dispersion damage parameters}

Rock damage is highly dependent on stress states. Once drilling into the formation to form a borehole, the heavier rock will be replaced by a much lighter drilling fluid, which will cause the redistribution of stress and the invasion of drilling fluid that disturbed by drilling operations. These phenomena will further cause the existing micro-cracks to propagate, connect, and even form macro-cracks or fractures until break down. Based on the theory of elastic mechanics, the rock anisotropy can be divided into intrinsic anisotropy and micro-cracks induced anisotropy, and the stress-strain relationship can expressed as (Gui et al. 2018):

$\varepsilon_{i j}=\left(S_{i j k l}^{o}+\Delta S_{i j k l}\right) \sigma_{k l}$

where $S_{i j k l}^{o}$ is the intrinsic compliance matrix; $\Delta S_{i j k l}$ is the micro-cracks induced compliance matrix.
Becker et al. (2007) conducted a series of theoretical and experimental examinations on the stress induced elastic anisotropy, and proposed a Swiss cheese model for layered rock materials. Shapiro and cooperator indicated that under the condition of high confining pressure, the normal and shear toughness of the contact area between clay particles is zero, and the stress interaction between crack contact surfaces can be ignored (Shapiro 2003; Shapiro and Kaselow 2005). As we know, the VTI formation rock in the vicinity of the wellbore is always subjected to high temperature and high pressure, in other words, the rock in the vicinity of the wellbore satisfies the assumptions of this model. Therefore, we assumed that the micro-cracks in the undisturbed VTI formation rock is in a closed state. Its compliance matrix is an inherent compliance matrix $S_{i j k l}^{o}$, while the variation of the elastic parameters in the vicinity of the wellbore is mainly caused by the microcracks opening under the influence of in situ environments and external operations. Sayers et al. (1990) proposed an additional second-order tensor $\alpha_{i j}$ and an additional fourthorder tensor $\beta_{i j k l}$ to express the micro-crack-induced compliance matrix $\Delta S_{i j k l}$ :

$\Delta S_{i j k l}=\frac{1}{4}\left(\delta_{i k} \alpha_{j l}+\delta_{i l} \alpha_{j k}+\delta_{j k} \alpha_{i l}+\delta_{j l} \alpha_{i k}\right)+\beta_{i j k l}$

where $\delta_{i k}$ is the Kronecker symbol; $\alpha_{i j}$ is an additional second-order tensor related to micro-cracks and pores; $\beta_{i j k l}$ is an additional fourth-order tensor related to pore fluids.

For tight sand formation, the compliance coefficients and stiffness coefficients meet the following relationship (Gui et al. 2018; Tang and Wu 2015):

$\left\{\begin{array}{l}S_{11}+S_{12}=C_{33} /\left[C_{33}\left(C_{11}+C_{12}\right)-2 C_{13}^{2}\right] \\ S_{11}-S_{12}=1 /\left(C_{11}-C_{12}\right) \\ S_{13}=-C_{13} /\left[C_{33}\left(C_{11}+C_{12}\right)-2 C_{13}^{2}\right] \\ S_{33}=\left(C_{11}+C_{12}\right) /\left[C_{33}\left(C_{11}+C_{12}\right)-2 C_{13}^{2}\right] \\ S_{13}=1 / C_{55}\end{array}\right.$

Due to the symmetry of VTI tensors $\left(\alpha_{i j}\right.$ and $\left.\beta_{i j k l}\right)$, the element of these two tensors $\left(\alpha_{i j}\right.$ and $\left.\beta_{i j k l}\right): \alpha_{11}=\alpha_{22}$, $\beta_{1111}=\beta_{2222}, \beta_{1212}=\beta_{1122}=\beta_{1111} / 3$. Therefore, according to Eqs. (11)-(13), the tensor elements can be solved using the compliance elements:

$$
\left\{\begin{array}{l}
\alpha_{11}=-2 \Delta S_{11}+1.5 \Delta S_{66} \\
\alpha_{33}=2 \Delta S_{33}+\Delta S_{44}-1.5 \Delta S_{66}-4 \Delta S_{13} \\
\beta_{1111}=3 \Delta S_{11}-1.5 \Delta S_{66} \\
\beta_{3333}=-2 \Delta S_{11}+\Delta S_{33}-\Delta S_{44}+1.5 \Delta S_{66}+4 \Delta S_{13} \\
\beta_{1133}=\Delta S_{13}
\end{array}\right.
$$


where $\alpha_{11}$ represents the vertical damage parameter; $\alpha_{33}$ represents the horizontal damage parameter.

It is obvious to find that the premise of evaluating rock damage is to calculate the additional compliance matrix induced by micro-cracks under the influence of in situ environments and external operations. The additional compliance matrix can be determined using the elastic stiffness parameter, while the elastic stiffness parameter can be determined using acoustic logging data. Thus, acoustic logging data can be utilized to evaluate the rock damage.

\section{Calculation method of radial distribution of parameters}

Although we had proposed the relationship between rock damage parameters and acoustic logging data, but we still cannot determine its radial distribution. As we know, the dipole shear wave logging instruments, such as the dipole shear sonic imager (DSI) and cross-multipole array acousti$\log$ (XMAC), can be used not only for testing S-wave velocities, but also for testing the dispersion of $\mathrm{S}$-wave velocities varying with the acoustic wave emission frequency at different radial distance points. In general, the S-wave velocities under different acoustic emission frequencies correspond to the S-wave response characteristics of rocks at different radial distance points (Sinha and Kostek 1996; Hornby 1993). Under the condition of high frequency acoustic emission, the vibration frequency of the logging instrument is fast, the wavelength of the excited S-wave is short, the propagation speed is slow, and the penetration ability is poor, while under the condition of low frequency acoustic emission, the vibration frequency of the logging instrument is slow, the excited $\mathrm{S}$-wave wavelength is long, the propagation speed is fast, and the penetration ability is relatively good, which reflects the acoustic response characteristics of the original formation (Sayers 1999; Tang and Patterson 2010). Thus, the radial section of the $\mathrm{S}$-wave velocity of the formation around the borehole wall can be obtained by processing the dispersion curve of each sampling radial distance.

For the acoustic wave excited by the dipole acoustic source, the S-wave waveform is a kind of curved wave with the characteristic of dispersion, and the equation of dispersion curve can be expressed as (Tang and Patterson 2010):

$D(k, w ; B, F(r))=0$

where $k$ is the wavenumber; $w$ is angular frequency; $B$ is the waveguide part of the hole, which is affected by the wellbore fluid and the logging instrument; $r$ is the radial distance; $F(r)$ is the velocity and density of the S-wave vary with the radial distance in an isotropic elastic formation.

Since the S-wave velocity and density change with the radial distance, the relationship between the $\mathrm{S}$-wave velocity and frequency can be obtained by solving Eq. (15), i.e., the $\mathrm{S}$-wave dispersion curve. The low frequency and high frequency characteristics of the dispersion curve are closely related to the S-wave velocity of the original and induced formation. In order to find out the corresponding relationship, it is necessary to construct an inversion model, so as to determine the velocity and thickness of the radial variation formation. The inversion equation is as follows (Tang and Patterson 2010; Su et al. 2013):

$$
\begin{aligned}
E(\Delta r, \Delta V)= & \sum_{\Omega}\left[V_{m}(\omega ; \Delta r, \Delta V)-V_{d}(\omega)\right]^{2} \\
& +\lambda \sum_{\Omega^{\prime}}\left[V_{m}(\omega ; \Delta r, \Delta V)-V_{h}(\omega)\right]^{2}
\end{aligned}
$$

where $V_{\mathrm{m}}$ is the dispersion curve function in terms of $\Delta r$ and $\Delta V$, and it is determined by Eq. (15); $\Delta r$ is the thickness of radial variation formation; $\Delta V$ is the change in $\mathrm{S}$-wave velocity of radial variation formation; $\Omega$ is the given frequency range of S-wave with a value of $3.5-10 \mathrm{kHz}$; is the sum of residuals for each frequency; $\Omega$ ' is the high frequency subset of the given frequency range with a value of $8-10 \mathrm{kH}$; $\lambda$ is a weight factor with a value of 2.0.

Once the radial velocity profile is obtained, the compliance matrix of the formation rock at different radial distance can be determined. Due to the assumption condition that the compliance matrix at infinitely distant equals to the intrinsic compliance matrix, in other words, the micro-cracks and pores in the formation rock far from the borehole are considered as the background medium and are inherent nature. But, for the formation rock in the vicinity of the wellbore, due to the redistribution of stress, the invasion of drilling fluid, and the disturbance of drilling operation, which will further cause the existing micro-cracks to propagate, connect, and even form macro-cracks or fractures until break down, by comparing the $\mathrm{S}$-wave velocity profile, the compliance matrix at any given distance can be determined. Thus, the above methods can be utilized to determine the distribution and evolution of rock mechanical properties, anisotropy and damage characteristics in the vicinity of the wellbore. Furthermore, it can provide some theoretical basis for in situ stress evaluation, borehole stability analysis and hydraulic fracturing design.

\section{Case study}

\section{Logging interpretation results of case well}

DL area is located in the south of the Ordos basin in China. The reservoir lithology is dense siltstone and fine sandstone, a typical kind of tight sand reservoir. The buried depth of the reservoir is $4000-4300 \mathrm{~m}$, the reservoir

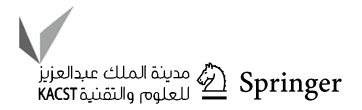


porosity ranges from 0.5 to $7 \%$, the hydrocarbon pore volume belongs to pore type. The dipole shear wave logging data and conventional well logging data collected from DL1 well were used to calculate the porosity, lithology, stiffness coefficients, elastic modulus and Poisson's ratio, and the results are shown in Fig. 1. From left to right, the first track is the measured depth, the second track is the borehole indications, the third track is the porosity indications, the fourth track is the lithology indications, the fifth track is the elastic parameters, and the sixth track is the rock mechanical parameters, and the final track is the comprehensive interpretation for hydrocarbons. The interpretation result for elastic parameters and rock mechanical parameters is the far-field original parameters that was not affected by the drilling disturbance. At a depth of $4200-4300 \mathrm{~m}$, the drill bit has a diameter of $9.5 \mathrm{in}$, but several sections showed an obvious borehole enlargement, such as $4200-4222 \mathrm{~m}$ and $4232-4250 \mathrm{~m}$. In order to eliminate the influence of borehole enlargement, only the non-enlargement section was selected for calculation. The results show that the order of the stiffness coefficients is roughly $C_{33}>C_{11}>C_{12} \approx C_{13}>C_{44} \approx C_{66}$, the transverse elastic modulus is significantly higher than that of longitudinal, and the longitudinal Poisson's ratio is significantly higher than that of the transverse, and the formation rock shows a very strong anisotropy characteristic. In addition, the elastic modulus is highly dependent on the sand content, the higher the sand content, the higher the elastic modulus, such as the section of 4215-4219 m and $4245-4265 \mathrm{~m}$, its elastic modulus is obviously higher than the other sections.

\section{Distribution of mechanical and damage parameters}

Although we had determined the rock mechanical parameters using acoustic logging data, we did not determine the damage parameters and its radial distribution. In order to distinguish the difference of sandstone and mudstone, two kinds of typical lithology (dominated by sandstone and pure mudstone) were identified to analyze and compare, such as the $4255 \mathrm{~m}$ (dominated by sandstone) and $4284 \mathrm{~m}$ (pure mudstone). Due to the limited detectivity of the dipole shear wave logging, its radial detectivity is usually within $1.0 \mathrm{~m}$, while the radial influencing distance of stress concentration is mainly within 4 times of hole radius. Thus, we just studied the changes of mechanical parameters, Thomsen's coefficients and damage parameters in the range of 4 times of hole radius.

\section{Sandstone in the depth of $4255 \mathrm{~m}$}

In the depth of $4255 \mathrm{~m}$, the formation lithology is mainly sandstone, and the original rock mechanical parameters explained by acoustic logging data are given as follows: $\mu_{v}=0.257, \mu_{h}=0.199, E_{v}=50.70 \mathrm{GPa}$, and $E_{h}=77.36 \mathrm{GPa}$. Compared with the horizontal direction, the vertical Poisson's ratio is much larger, while the vertical elastic modulus is much smaller, this is due to the compact effect. The anisotropic coefficients of $\mathrm{P}$-wave and S-wave are $\varepsilon=-0.039$ and $\gamma=-0.057$, respectively, which means that the anisotropy of $\mathrm{S}$-wave is much greater than that of $\mathrm{P}$-wave.

Figure 2 shows the radial distribution of rock mechanical parameters. It has obviously been noticed that both vertical and horizontal elastic modulus $\left(E_{v}\right.$ and $\left.E_{h}\right)$ decreased in the vicinity of the wellbore, while both vertical and horizontal Possion's ratios $\left(\mu_{v}\right.$ and $\left.\mu_{h}\right)$ increased. But the vertical elastic modulus still lower than that of the horizontal, while the vertical Possion's ratio still greater than that of the horizontal. The elastic modulus nonlinearly increased with radial distance, while the Possion's ratio nonlinearly decreased, and the influencing degree gradually diminish with increasing in radial distance. However, the growth of vertical elastic modulus is significantly greater than that of the horizontal, and its growing proportion for vertical and horizontal is 2.05 and $0.66 \%$; the decline of vertical Possion's ratio is slightly greater than that of the horizontal, but its decline proportion for vertical and horizontal is 1.85 and $2.22 \%$, due to the much lower original value of horizontal Possion's ratio. The main influencing range of mechanical parameters changing is within 2.5 times of hole radius, and all mechanical parameters rapidly decreased in the range of $1.0-1.8$ times of hole radius.

Figure 3 shows the radial distribution of Thomsen's coefficients. It has obviously been noticed that the anisotropic coefficients of both P-wave and S-wave ( $\varepsilon$ and $\gamma$ ) increased with radial distance. In other words, the degree of anisotropy was lowered for both P-wave and S-wave in the vicinity of the wellbore, which may therefore make the logging interpretation results incorrect, especially for highly anisotropy-dependent logging interpretations, such as elastic parameters, Thomsen's coefficients, in situ stress, and fracture pressure. The degree of anisotropy for P-wave is obviously greater than that of S-wave. The decline magnitude of P-wave anisotropic coefficient is significantly greater than that of $\mathrm{S}$-wave, but its decline proportion of $\mathrm{P}$-wave and $\mathrm{S}$-wave is 54.22 and $56.39 \%$, due to the much higher original value of anisotropic coefficients for P-wave. The main influencing range of Thomsen's coefficients changing is within 2.8 times of hole radius, and all Thomsen's coefficients rapidly decreased in the range of 1.0-1.8 times of hole radius. 


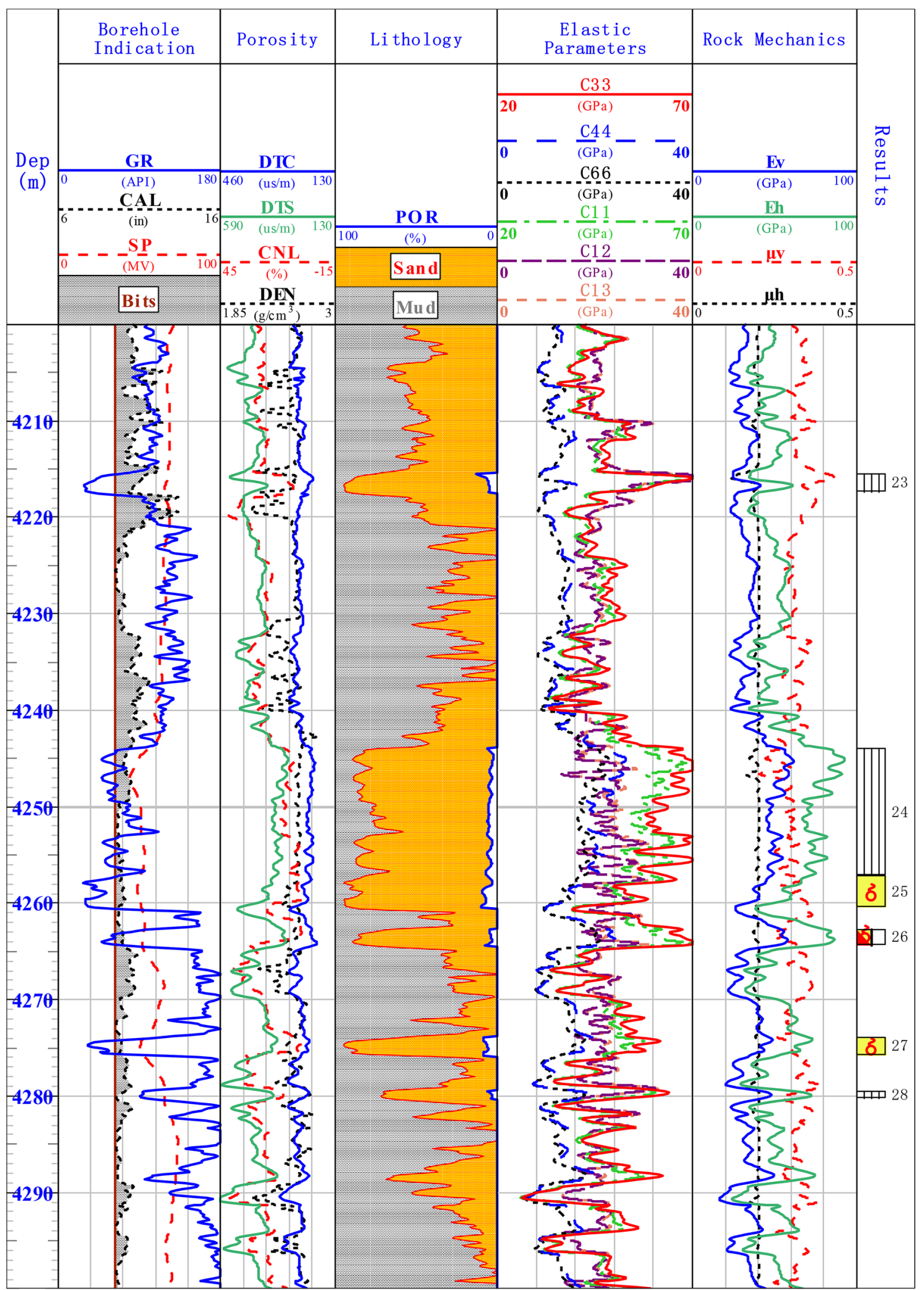

Fig. 1 Logging interpretation results of DL1 well 


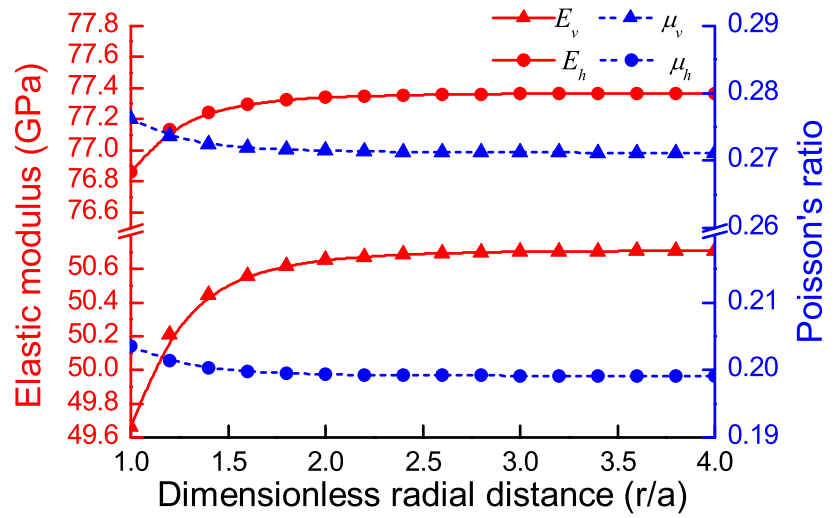

Fig. 2 Distribution of mechanical parameters for sandstone

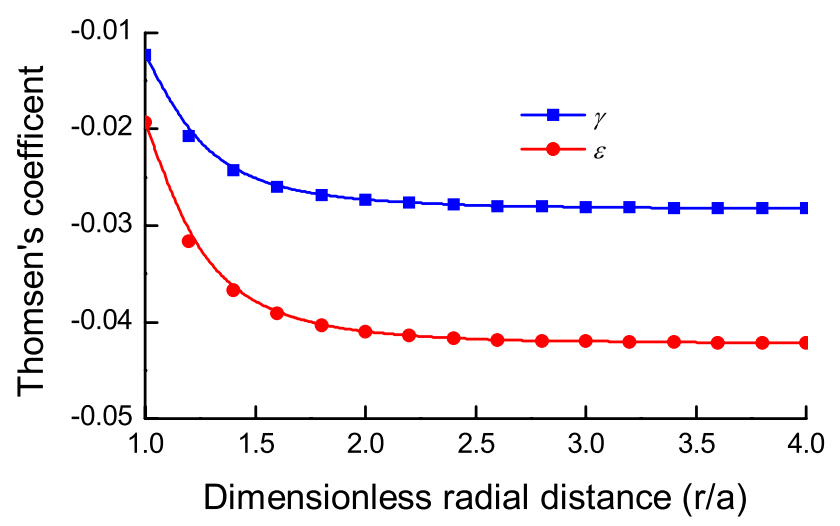

Fig. 3 Distribution of Thomsen's coefficients for sandstone

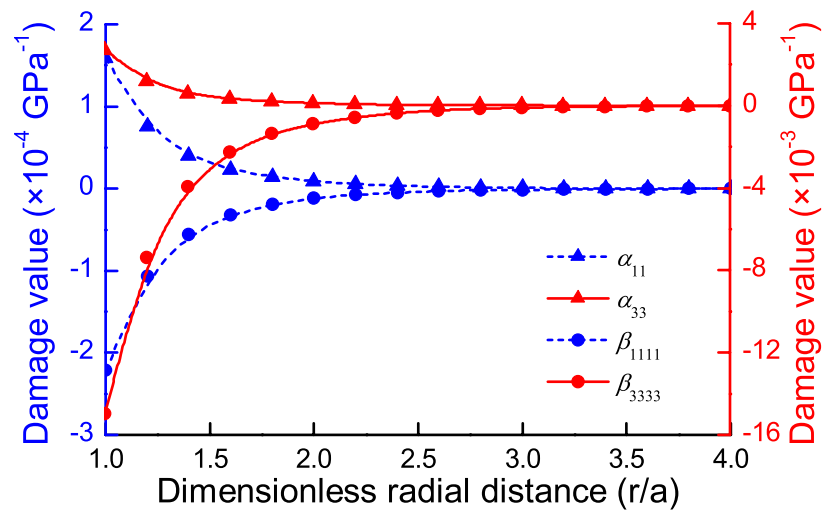

Fig. 4 Distribution of damage parameters for sandstone

Figure 4 shows the radial distribution of rock damage parameters. It has obviously been noticed that both vertical and horizontal damage parameters decreased with the radial distance, and the induced damage of $\alpha_{11}$ increased to $1.59 \times 10^{-4} \mathrm{GPa}, \alpha_{33}$ increased to $2.69 \times 10^{-3} \mathrm{GPa}$,

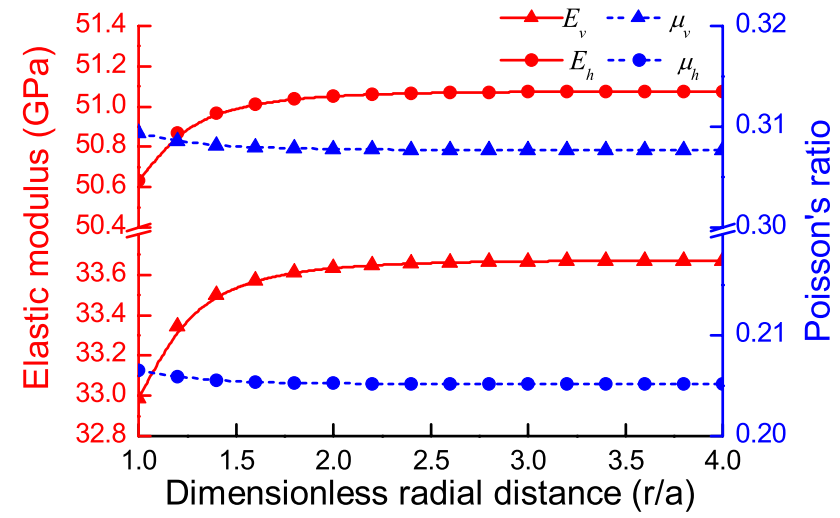

Fig. 5 Distribution of mechanical parameters for mudstone

$\beta_{1111}$ increased to $-2.22 \times 10^{-4} \mathrm{GPa}, \beta_{3333}$ increased to $-1.50 \times 10^{-2} \mathrm{GPa}$. In other words, the damage parameters increased in the vicinity of the wellbore, due to the redistribution of stress, the invasion of drilling fluid, and the disturbance of drilling operation. The horizontal damage parameter $\left(\alpha_{33}\right)$ is obviously greater than that of the vertical $\left(\alpha_{11}\right)$, and the horizontal damage parameter $\left(\alpha_{33}\right)$ is appropriately 17 times of the vertical damage parameter $\left(\alpha_{11}\right)$. Thus, the rock damage induced by micro-cracks, stresses and wellbore operations mainly occurred in the horizontal direction. The main influencing range of rock damage is within 2.8 times of hole radius, when the radial distance reaches the 2.8 times of hole radius, there is almost no impact on damage parameters. In addition, all damage parameters rapidly decreased in the range of 1.0-1.8 times of hole radius, and the wellbore enlargement occurred at the depth of $4218 \mathrm{~m}$ just within this range.

\section{Mudstone in the depth of $4284 \mathrm{~m}$}

In the depth of $4284 \mathrm{~m}$, the formation lithology is mainly mudstone, and the original rock mechanical parameters explained by acoustic logging data are given as follows: $\mu_{v}=0.308, \mu_{h}=0.205, E_{v}=33.67 \mathrm{GPa}$, and $E_{h}=51.07 \mathrm{GPa}$. Compared with the horizontal direction, the vertical Poisson's ratio is much larger, while the vertical elastic modulus is smaller, this is also due to the compaction effect. The anisotropic coefficients of $\mathrm{P}$-wave and $\mathrm{S}$-wave are $\varepsilon=-0.010$ and $\gamma=-0.017$, respectively, which means that the anisotropy of $\mathrm{S}$-wave is much greater than that of $\mathrm{P}$-wave.

Figure 5 shows the radial distribution of rock mechanical parameters. It has obviously been noticed that both vertical and horizontal elastic modulus $\left(E_{v}\right.$ and $\left.E_{h}\right)$ decreased in the vicinity of the wellbore, while both vertical and horizontal Possion's ratios $\left(\mu_{v}\right.$ and $\left.\mu_{h}\right)$ increased. But the vertical elastic modulus still lower than that of the horizontal, while the vertical Possion's ratio still greater than that of 
the horizontal. The elastic modulus nonlinearly increased with radial distance, while the Possion's ratio nonlinearly decreased, and the influencing degree gradually diminish with increasing in radial distance. However, the growth of vertical elastic modulus is significantly greater than that of the horizontal, and its growing proportion for vertical and horizontal is 2.03 and $0.86 \%$; the decline of vertical Possion's ratio is slightly greater than that of horizontal, but its decline proportion for vertical and horizontal is 0.54 and $0.65 \%$, due to the much lower original value of horizontal Possion's ratio. The main influencing range of mechanical parameters changing is within 2.5 times of hole radius, and all mechanical parameters rapidly decreased in the range of $1.0-1.8$ times of hole radius.

Figure 6 shows the radial distribution of Thomsen's coefficients. It has obviously been noticed that the anisotropic coefficients of both P-wave and S-wave ( $\varepsilon$ and $\gamma$ ) increased with radial distance. In other words, the degree of anisotropy was lowered for both P-wave and S-wave in the vicinity of the wellbore. However, the degree of anisotropy was lowered to 0 at the radial distance of 1.3 times of hole radius, but it is continually reverse growth within 1.3 times of hole radius, and the reversed degree of anisotropy can reach to the original degree at the radial distance of 1.5 times of hole radius, which may therefore make the logging interpretation results incorrect. The change magnitude of P-wave anisotropic coefficient is significantly greater than that of $\mathrm{S}$-wave, and its proportion is 165.36 and $161.84 \%$ for P-wave and S-wave, respectively. The main influencing range of Thomsen's coefficients changing is still within 2.8 times of hole radius, and all Thomsen's coefficients rapidly decreased in the range of $1.0-1.8$ times of hole radius.

Figure 7 shows the radial distribution of rock damage parameters. It has obviously been noticed that both vertical and horizontal damage parameters decreased with the radial distance, and the induced damage of $\alpha_{11}$ increased to $1.91 \times 10^{-4} \mathrm{GPa}, \alpha_{33}$ increased to $6.49 \times 10^{-3} \mathrm{GPa}$,

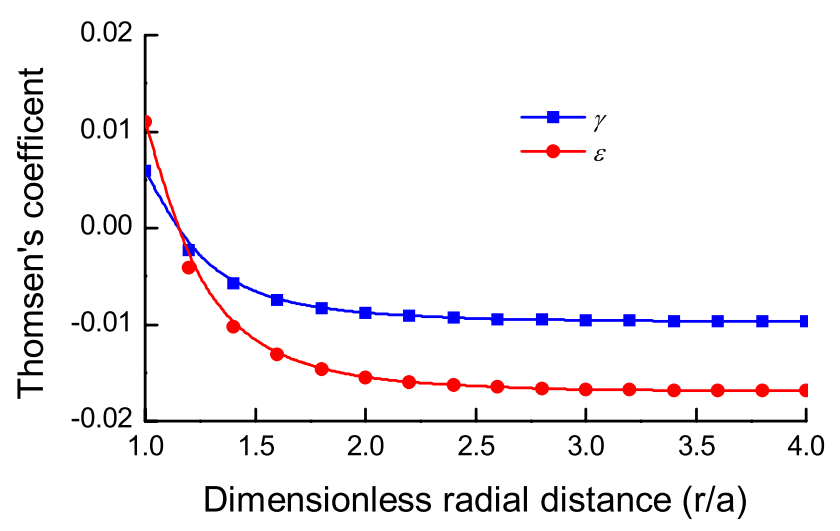

Fig. 6 Distribution of Thomsen's coefficients for mudstone

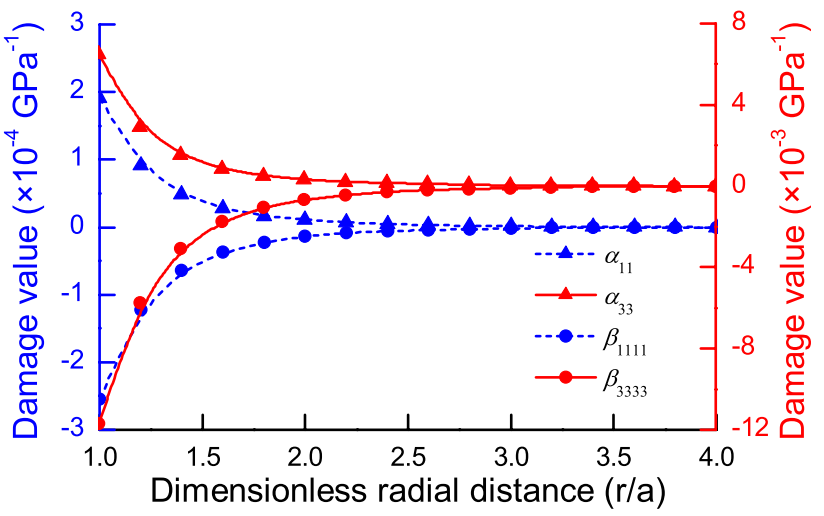

Fig. 7 Distribution of damage parameters for mudstone

$\beta_{1111}$ increased to $-2.59 \times 10^{-4} \mathrm{GPa}, \beta_{3333}$ increased to $-1.17 \times 10^{-2} \mathrm{GPa}$. In other words, the damage parameters increased in the vicinity of the wellbore, due to the redistribution of stress, the invasion of drilling fluid, and the disturbance of drilling operation. The horizontal damage parameter $\left(\alpha_{33}\right)$ is obviously greater than that of the vertical $\left(\alpha_{11}\right)$, and the horizontal damage parameter $\left(\alpha_{33}\right)$ is appropriately 34 times of the vertical damage parameter $\left(\alpha_{11}\right)$. Thus, the rock damage induced by micro-cracks, stresses and wellbore operations mainly occurred in the horizontal direction. The main influencing range of rock damage is within 2.8 times of hole radius, and all damage parameters rapidly decreased in the range of 1.0-1.8 times of hole radius.

\section{Comparison between sandstone and mudstone}

Based on the calculation results of rock mechanical parameters, Thomsen's coefficients and damage parameters at two depths of 4255 and $4284 \mathrm{~m}$, it is obviously found that with increasing radial distance, the Poisson's ratio decreased, the elastic modulus increased, the Thomsen's coefficients increased, but the damage parameters decreased. For both sandstone and mudstone, the vertical Poisson's ratio is much larger than that of horizontal, but the vertical elastic modulus is smaller than that of horizontal; the degree of anisotropy for P-wave is obviously greater than that of S-wave; the horizontal damage parameter is obviously greater than that of vertical, which indicated that the micro-cracks near the wellbore mainly occur in the horizontal direction. Almost all of the changes in mechanical parameters, Thomsen's coefficients and damage parameters mainly occurred in the scope of 1.0-2.8 times of hole radius, and all parameters rapidly changed in the range of 1.0-1.8 times of hole radius. However, there are some difference between sandstone and mudstone, the original elastic modulus of sandstone is obviously much higher than mudstone, the original Poisson's ratio of sandstone is slightly lower than mudstone, the original degree of anisotropy for sandstone is more significant than mudstone. The variations of mudstone

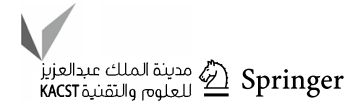


in Thomsen's coefficients and damage parameters are obviously greater than that of sandstone, this may be due to the mudstone is much looser than sandstone, which makes its influencing scope of stress concentration is not as large as that of sandstone, but because of the interaction between mudstone and drilling fluid, the induced damage of mudstone is much higher than sandstone.

In addition, the limitation of the present method can be concluded as follows: (1) The present model just can suit for vertically transverse isotropic (VTI) medium, which means it just can suit for horizontal formation. If the formation is inclined, the present model cannot suit for inclined transverse isotropic medium. (2) The original rock stiffness coefficients are the basis of calculation for radial distribution of damage parameter; however, due to the limitation of current full wave logging technology, only three stiffness coefficients $\left(C_{33}, C_{44}\right.$, and $\left.C_{66}\right)$ can be obtained directly by using full wave logging data (Tang and Zheng 2004; Wang et al. 2007), while the other stiffness coefficients $\left(C_{11}, C_{12}\right.$, and $C_{13}$ ) were determined by using empirical equations (Schoenberg et al. 1996; Schoenberg and Douma 1998; Sayers 2008). (3) Regarding the dispersion damage parameters, combined with Eshelby's tensor (Eshelby 1957) and the elliptical porosity model in TI media (Withers 1989), the pores and the matrix were treated as a whole background, the circular pores and fractured pores were added into the background (Sarout and Guéguen 2008; Gui et al. 2018). The circular pores contribute considerably to the total porosity, but they are not sensitive to the stress, whereas the fractured pores, which are "lying" in the bedding plane of the rock (Sarout and Guéguen 2008), are extremely sensitive to the triaxial stress and can reflect the variation of the elastic wave velocity (Gui et al. 2018). Based on the above theory, the dispersion damage parameters are determined by the additional compliance matrix induced by micro-cracks under the influence of in situ environments and external operations. (4) Regarding the calculation method of radial distribution of parameters, it is affected by the wellbore fluid and the logging instrument, the equation of dispersion curve is very important aspect to determine the radial distribution of the parameters.

\section{Conclusions}

In this paper, the damage characteristics in the vicinity of the wellbore were evaluated by using acoustic logging data interpretation, the formation medium was regarded as a VTI medium, combined with a dispersion damage model, dipole shear wave logging data and inversion method of radial wave velocity profile, the original and changed mechanical parameters, Thomsen's coefficients and damage parameters were calculated and analyzed. Through the research in this paper, the following main conclusions can be drawn:

1. With increasing in radial distance, the Poisson's ratio decreased, while the elastic modulus increased. The vertical Poisson's ratio is much larger than that of horizontal, while the vertical elastic modulus is smaller than that of the horizontal. With increasing in radial distance, the Thomsen's coefficients increased, and the degree of anisotropy for P-wave is obviously greater than that of $\mathrm{S}$-wave. Almost all of the changes in mechanical parameters and Thomsen's coefficients mainly occurred in the scope of 2.5-2.8 times of hole radius, and all parameters rapidly changed in the range of 1.0-1.8 times of hole radius.

2. With increasing in radial distance, the damage parameters decreased, the changes in damage parameters mainly occurred in the scope of 2.5-2.8 times of hole radius, all parameters rapidly changed in the range of $1.0-1.8$ times of hole radius, and the horizontal damage parameter is obviously greater than that of vertical.

3. The original elastic modulus of sandstone is obviously much higher than mudstone, the original Poisson's ratio of sandstone is slightly lower than mudstone, and the original degree of anisotropy for sandstone is more significant than mudstone. The variations of mudstone in Thomsen's coefficients and damage parameters are obviously greater than that of sandstone.

Funding This work was supported by the Sichuan Science and Technology Program (Grant No. 2020JDJQ0055), the Fok Ying-Tong Education Foundation, China (Grant No. 171097), the National Natural Science Foundation of China (Grant No. 41874216), the Youth Scientific and Technological Innovation Team Foundation of Southwest Petroleum University (Grant No. 2019CXTD09).

\section{Declarations}

Conflicts of interest The authors have no conflicts of interest to declare that are relevant to the content of this article.

Open Access This article is licensed under a Creative Commons Attribution 4.0 International License, which permits use, sharing, adaptation, distribution and reproduction in any medium or format, as long as you give appropriate credit to the original author(s) and the source, provide a link to the Creative Commons licence, and indicate if changes were made. The images or other third party material in this article are included in the article's Creative Commons licence, unless indicated otherwise in a credit line to the material. If material is not included in the article's Creative Commons licence and your intended use is not permitted by statutory regulation or exceeds the permitted use, you will need to obtain permission directly from the copyright holder. To view a copy of this licence, visit http://creativecommons.org/licenses/by/4.0/. 


\section{References}

Becker K, Shapiro SA, Stanchits S, Dresen G, Vinciguerra S (2007) Stress induced elastic anisotropy of the Etnean basalt: Theoretical and laboratory examination. Geophys Res Lett 34(34):224-238

Boosari SSH, Aybar U, Eshkalak MO (2016) Unconventional resource's production under desorption-induced effects. Petroleum 2(2): 148-155

Chai Y, Wang G, Zhang X, Ran Y (2016) Pore structure characteristics and logging recognition of tight sandstone reservoir of the second member of Xujiahe Formation in Anyue Area, central Sichuan. J Cent South Univ (Sci Technol) 47(3):819-827

Chaki S, Takarli M, Agbodjan WP (2008) Influence of thermal damage on physical properties of a granite rock: porosity, permeability and ultrasonic wave evolutions. Constr Build Mater 22(7):1456-1461

Chang SH, Lee CI (2004) Estimation of cracking and damage mechanisms in rock under triaxial compression by moment tensor analysis of acoustic emission. Int J Rock Mech Min Sci 41(7):1069-1086

Cheng X (2019) Damage and failure characteristics of rock similar materials with pre-existing cracks. Int J Coal Sci Technol 6:505-517

Chu CQ, Wu SC, Zhang SH, Guo P, Zhang M (2020) Mechanical behavior anisotropy and fracture characteristics of bedded sandstone. J Cent South Univ (Sci Technol) 51(8):2232-2246

Dai JX, Ni YY, Wu XQ (2012) Tight gas in China and its significance in exploration and exploitation. Pet Explor Dev 39(3):257-264

Denney D (2012) Improving horizontal completions in heterogeneous tight shales. J Petrol Technol 64(10):126-130

Eberhardt E, Stead D, Stimpson B (1999) Quantifying progressive prepeak brittle fracture damage in rock during uniaxial compression. Int J Rock Mech Min Sci 36(3):361-380

Eshelby JD (1957) The determination of the elastic field of an ellipsoidal inclusion, and related problems. Proceedings of the Royal Society of London. Series A. Mathematical and physical sciences, 241, 376-396

Gui JC, Ma TS, Chen P, Yuan HY, Guo ZX (2018) Anisotropic damage to hard brittle shale with stress and hydration coupling. Energies 11(4):926

Gui JC, Ma TS, Chen P (2020) Rock physics modeling of transversely isotropic shale: an example of the Longmaxi formation in the Sichuan basin. Chin J Geophys 63(11):4188-4204

Guo JC, Gou B (2015) Design philosophy and practice of asymmetrical 3D fracturing and random fracturing: a case study of tight sand gas reservoirs in western Sichuan Basin. Nat Gas Ind B 2(2-3): 174-180

Higgins S, Goodwin S, Donald A, Bratton TR, Tracy GW (2008) Anisotropic stress models improve completion design in the Baxter Shale. In: SPE Annual Technical Conference and Exhibition, 21-24 September, Denver, Colorado, USA.

Hornby BE (1993) Tomographic reconstruction of near-borehole slowness using refracted borehole sonic arrivals. Geophysics 58(12): 1726-1738

Huang L, Liu X, Yan S, Xiong J, He H, Xiao P (2020) Experimental study on the acoustic propagation and anisotropy of coal rocks. Petroleum. https://doi.org/10.1016/j.petlm.2020.10.004

Liu Y, Ma TS, Wu H, Chen P (2020) Investigation on mechanical behaviors of shale cap rock for geological energy storage by linking macroscopic to mesoscopic failures. J Energy Storage 29:101326

Lu T, Liu YX, Wu LC, Wang XW (2015) Challenges to and countermeasures for the production stabilization of tight sandstone gas reservoirs of the Sulige Gasfield. Ordos Basin Nat Gas Ind B 2(4):323-333
Luo C, Jia AL, Guo JL, He DB, Wei YS, Luo SL (2016) Analysis on effective reservoirs and length optimization of horizontal wells in the Sulige Gasfield. Nat Gas Ind B 3(3):245-252

Luo Z, Zhang N, Zhao L, Yuan X, Zhang Y (2018) A novel stimulation strategy for developing tight fractured gas reservoir. Petroleum 4(2):215-222

Ma TS, Chen P (2014) Study of meso-damage characteristics of shale hydration based on CT scanning technology. Pet Explor Dev 41(2):249-256

Ma TS, Peng N, Chen P, Yang CH, Wang XM, Han X (2018) Study and verification of a physical simulation system for formation pressure testing while drilling. Geofluids 2018:1-18

Ma TS, Yang CH, Chen P, Wang XD, Guo YT (2016) On the damage constitutive model for hydrated shale using CT scanning technology. J Nat Gas Sci Eng 28:204-214

Mavko G, Mukerji T, Godfrey N (1995) Predicting stress-induced velocity anisotropy in rocks. Geophysics 60(4):1081-1087

Małkowski P, Niedbalski Z, Balarabe T (2020) A statistical analysis of geomechanical data and its effect on rock mass numerical modeling: a case study. Int J Coal Sci Technol. https://doi.org/10.1007/ s40789-020-00369-2

Ming H, Sun W, Zhang L, Wang Q (2015) Impact of pore structure on physical property and occurrence characteristics of moving fluid of tight sandstone reservoir: taking $\mathrm{He} 8$ reservoir in the east and southeast of Sulige gas field as an example. J Cent South Univ (Sci Technol) 46(12):4556-4567

Sarout J, Molez L, Guéguen Y, Hoteit N (2007) Shale dynamic properties and anisotropy under triaxial loading: experimental and theoretical investigations. Phys Chem Earth, Parts a/b/v 32(8-14):896-906

Sarout J, Guéguen Y (2008) (2008) Anisotropy of elastic wave velocities in deformed shales: Part 2-Modeling results. Geophysics 73:D91-D103

Sayers CM (1999) Stress-dependent seismic anisotropy of shales. Geophysics 64(1):93-98

Sayers CM (2008) The effect of low aspect ratio pores on the seismic anisotropy of shales. In: SEG Technical Program Expanded Abstracts, pp 2750-2754. https://doi.org/10.1190/1.3063916

Sayers CM, Kachanov M (1995) Microcrack-induced elastic wave anisotropy of brittle rocks. J Geophys Res: Solid Earth 100(B3):4149-4156

Sayers CM, Munster JGV, King MS (1990) Stress-induced ultrasonic anisotrophy in Berea sandstone. Int J Rock Mech Min Sci Geomech Abstr 27(5):429-436

Schoenberg M (1980) Elastic wave behavior across linear slip interfaces. J Acoust Soc Am 68(5):1516-1521

Schoenberg M, Douma J (1998) Elastic wave propagation in media with parallel fractures and aligned cracks. Geophys Prospect 36(6):571-590

Schoenberg M, Muir F, Sayers C (1996) Introducing ANNIE: a simple three-parameter anisotropic velocity model for shales. J Seism Explor 5(1):35-49

Shapiro SA (2003) Elastic piezosensitivity of porous and fractured rocks. Geophysics 68(2):482-486

Shapiro SA, Kaselow A (2005) Porosity and elastic anisotropy of rocks under tectonic stress and pore-pressure changes. Geophysics 70(5):27-38

Sinha BK, Kostek S (1996) Stress-induced azimuthal anisotropy in borehole flexural waves. Geophysics 61(6):1899-1907

Song Z, Ji H, Liu Z, Sun L (2020) Study on the critical stress threshold of weakly cemented sandstone damage based on the renormalization group method. Int J Coal Sci Technol 7:693-703

Su YD, Tang XM, Zhuang CX, Xu S, Zhao L (2013) Mapping formation shear-velocity variation by inverting logging-while-drilling quadrupole-wave dispersion data. Geophysics 78(6):D491-D498

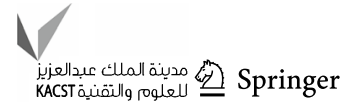


Tang J, Wu GC (2015) Stress-dependent anisotropy of mudstone and shale with low porosity. Chin J Geophys 58(8):2986-2995

Tang XM, Patterson DJ (2010) Mapping formation radial shear-wave velocity variation by a constrained inversion of borehole flexuralwave dispersion data. Geophysics 75(6):E183-E190

Tang XM, Zheng CH (2004) Quantitative acoustic logging. Petroleum Industry Press, Beijing, p 208

Thomsen L (1986) Weak elastic anisotropy. Geophysics 51(10):1954-1966

Walsh J, Sinha B, Donald A (2006) Formation anisotropy parameters using borehole sonic data. In: SPWLA 47th Annual Logging Symposium, 4-7 June, Veracruz, Mexico

Wang H, Wang B, Jing AY, Tao G (2007) An algorithm for extracting shear-wave anisotropy parameter $\gamma$ from stoneley-wave inversion and its case study. Well Logging Technol 31(3):241-244

Wang RJ, Qiao WX, Ju XD (2012) Numerical study of formation anisotropy evaluation using cross dipole acoustic LWD. Chin J Geophys 55(11):3870-3882

Wang XZ, Qiao XY, Mi NZ, Wang RG (2019) Technologies for the benefit development of low-permeability tight sandstone gas reservoirs in the Yan'an Gas Field. Ordos Basin Nat Gas Ind B 6(3):272-281

Wang T, Ju B, Wang S, Yang Z, Liu Q (2020) A tight sandstone multiphysical hydraulic fractures simulator study and its field application. Petroleum 6(2):198-205

Withers PJ (1989) The determination of the elastic field of an ellipsoidal inclusion in a transversely isotropic medium, and its relevance to composite materials. Philos Mag A 59(4):759-781

Xue L, Qin SQ, Sun Q, Wang YY, Lee LM, Li WC (2014) A study on crack damage stress thresholds of different rock types based on uniaxial compression tests. Rock Mech Rock Eng 47(4):1183-1195
Xie ZQ, Zhang YC, Xiao HB, Fan JZ (2012) Acoustic full wave logging of karst foundation grouting effect evaluation. J Cent South Univ (Sci Technol) 43(7):2757-2761

Xiong J, Liu K, Liu X, Liang L, Zhang C (2021) Influences of bedding characteristics on the acoustic wave propagation characteristics of shales. Petroleum 7(1):33-38

Yang X, Weng L, Hu Z (2018) Damage evolution of rocks under triaxial compressions: an NMR investigation. KSCE J Civ Eng 22(8):2856-2863

Yang X, Shi X, Meng Y, Xie X (2020) Wellbore stability analysis of layered shale based on the modified Mogi-Coulomb criterion. Petroleum 6(3):246-252

Zhang Z, Zhang R, Xie H, Liu J, Were P (2015) Differences in the acoustic emission characteristics of rock salt compared with granite and marble during the damage evolution process. Environ Earth Sci 73(11):6987-6999

Zhou JH, Yang K, Fang K, Zhao TB, Qiu DW (2019) Effect of fissure on mechanical and damage evolution characteristics of sandstone containing hole defect. J Cent South Univ (Sci Technol) 50(4):968-975

Zhu ZN, Jiang GS, Tian H, Wu WB, Liang RZ, Dou B (2019) Study on statistical thermal damage constitutive model of rock based on normal distribution. J Cent South Univ (Sci Technol) 50(6):1411-1418

Zou CN, Yang Z, He DB, Wei YS, Li J, Jia AL, Chen JJ, Zhao Q, Li YL, Li J, Yang S (2018) Theory, technology and prospects of conventional and unconventional natural gas. Pet Explor Dev 45(4):575-587

Publisher's Note Springer Nature remains neutral with regard to jurisdictional claims in published maps and institutional affiliations. 